\title{
Escuelas Superiores de Ciencias Aplicadas: Un primer caso de estudio
}

\author{
Multidisciplinary Research and Education for the Reduction of \\ Migration Needs: A first case study
}

Rainer Christoph* Master in Physical Chemistry, University of Bern Correo electrónico: rainer@nanotecnia.net

Ángel Hernández
Ingeniero en Sistemas Informáticos, Universidad de E1 Salvador
aahernandez@ufg.edu.sv
Romeo Muñoz
Ingeniero en Sistemas, Universidad de E1 Salvador
ramunoz@ufg.edu.sv
Jonathan Ventura
Estudiante Ingeniería Industrial, Universidad Francisco Gavidia
jventura@ufg.edu.sv
Wolfgang Büscher
Mechanical Engineering, Technical University Munich, Germany
wolfgang.buescher@googlemail.com

Todos los autores trabajan en el Laboratorio de Nanotecnología de la Universidad Francisco Gavidia

* Autor principal

Recibido: 4 de octubre de 2019 Aprobado: 30 de noviembre de 2019

DOI: $10.5377 /$ ryr.v50i50.9098

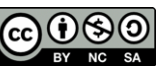




\section{RESUMEN}

La investigación aplicada multidisciplinaria, combinada con enfoques educativos modernos representan una potente herramienta para buscar y desarrollar soluciones a la medida que mejoran las condiciones de vida de amplios sectores de la población de Centro América, aportando también a reducir la necesidad de tener que migrar.

En este estudio se combinaron modernas metodologías de investigación aplicada, i.e. las Nanotecnologías, con un enfoque de educación multidisciplinario y orientado en la solución de problemas, i.e. la metodología STEM, para buscar soluciones innovadoras y sostenibles para necesidades básicas como agua, seguridad alimentaria, refrigeración y reducción de la violencia escolar.

Basado en los alentadores resultados obtenidos, proponemos la creación de una red Centroamericana de Escuelas Superiores de Investigación Aplicada, dedicadas a desarrollar soluciones innovadoras, basadas en materiales y recursos locales disponibles, para resolver necesidades básicas y específicas de las poblaciones de cada región.

Palabras clave: Educación STEM, Investigación aplicada, Nanotecnología, Desalinización, Nano-agricultura, Enfriamiento radiativo.

\section{ABSTRACT}

Multidisciplinary applied research combined with modern educational approaches represent a powerful tool for seeking and developing tailor-made solutions that improve the living conditions of broad sectors of the population of Central America, thus reducing their needs to migrate.

In this stud, wey combine modern applied research methodologies, i.e. Nanotechnologies, with a multidisciplinary, problem-solving approach to education, i.e. the STEM methodology, to seek innovative and sustainable solutions to basic needs such as safe water, food security, refrigeration and school violence reduction.

Based on the encouraging results obtained, we propose the creation of a Central American network of Applied Research Colleges, dedicated to developing innovative solutions, based on available local materials and resources, to solve basic and specific needs of the populations of each region.

Key words: STEM Education, Applied research, nanotechnology, desalination, nano-agriculture, radiative cooling.

\section{Introducción}

El abastecimiento sustentable con recursos básicos como agua, alimentación, vivienda, salud ambiental y educación es un importantísimo prerrequisito para garantizar una digna calidad de vida de las poblaciones y evitar flujos migratorios masivos en la región de Mesoamérica, pero también en otras regiones del mundo (Organización de las Naciones Unidas para la Alimentación y la Agricultura-FAO-, OPS, WFP, OMS, \& UNICEF, 2018).

\section{La necesidad de cubrir estas necesidades en cantidad} y calidad idónea se vuelve aún más importante en vista de impactos consecuentes del cambio climático (Banco Interamericano de Desarrollo, 2016). Consecuentemente, la generación de soluciones innovadoras y eficientes para enfrentar este enorme desafío también representa uno de los mayores retos para la ciencia y la tecnología.

Como condicionante fundamental de su sustentabilidad, estas soluciones innovadoras tienen que ser i) energéticamente optimizadas y viables, y ii) no-contaminantes, lo que básicamente se traduce a estar basadas en recursos, tanto humanos como materiales, localmente disponibles en las regiones afectadas y en cantidades suficientes. 
Otro factor importante es la gran complejidad de los temas investigativos necesarios para buscar y encontrar dichas innovaciones $y$ soluciones. Esta segunda condicionante exige utilizar enfoques multidisciplinarios, donde se combinan las ciencias, tanto en sus disciplinas naturales tradicionales (química, física, biología) como humanísticas (sociología, antropología, psicología, ética), con disciplinas de ingeniería (i.e. matemáticas, mecánica, hidráulica y eléctrica), y el uso de tecnologías modernas avanzadas de materiales, información y comunicación. La necesidad de utilizar este enfoque multidisciplinario sin duda también representa nuevos retos en el ámbito educativo.

En un primer intento de aplicar el concepto anteriormente descrito, hemos concentrado nuestros esfuerzos en el desarrollo local de capacidades innovadoras de investigación y educación multidisciplinaria, de manera práctica, y enfocada desde su inicio a la búsqueda de soluciones para fortalecer el abastecimiento de recursos básicos para la población del país.

Mediante una estrecha colaboración con la Escuela de Ingeniería de la Universidad San Carlos, Guatemala, así como el Laboratorio Nacional de Nanotecnología de Costa Rica hemos aplicado un enfoque moderno $\mathrm{y}$ multidisciplinario de ciencia y tecnología, i.e. la Nanotecnología (Bhushan, 2004) para identificar, y en algunos casos desarrollar, soluciones innovadoras, basadas en la utilización de recursos locales, para:

- Purificar y desalinizar aguas contaminadas y/o salobres con energías renovables y materiales innovadores.
- Reducir la vulnerabilidad de cultivos ante la escasez de agua y nutrientes, mediante la utilización de hidrogeles y nano-suspensiones.

- Conservar alimentos mediante nuevas técnicas de enfriamiento radiativo pasivo.

- Reducir la propagación de enfermedades infectocontagiosas mediante el uso de superficies auto-desinfectantes con propiedades foto-catalíticas.

- Mitigar la vulnerabilidad ante las consecuencias medioambientales que conllevan desechos de productos tecnológicos importados de uso masivo, mediante la reconversión de desechos procesos pirolíticos.

En el ámbito educativo, nos hemos enfocado en la implementación de nuevas metodologías de enseñanza con un enfoque holístico al aprendizaje de ciencias, tecnologías, ingenierías y matemática, y con fuerte orientación de educar en solucionar problemas, como es el caso para la metodología STEM (Rodger W. Bybee, 2013).

A continuación, presentamos un breve resumen de las metodologías utilizadas, principales resultados obtenidos, y nuestras conclusiones.

\section{Metodología}

Como principales metodologías utilizadas para esta investigación se ha enfocado en la Nanotecnología y la Educación STEM.

\section{Nanotecnología}

La Nanotecnología (NT) representa un esfuerzo colectivo para diseñar, caracterizar, 
producir y aplicar estructuras, dispositivos y sistemas mediante el control de la forma y el tamaño a escalas nanómetricas $\left(10^{-9} \mathrm{~m}\right)$ (Dowling et al., 2004).

La reciente aparición y proliferación de las Nanociencias y las Nanotecnologías (NCyNT) se ha visto favorecida por una serie de descubrimientos e invenciones fundamentales, como i) la conciencia física de que la fabricación de dispositivos nano-estructurados es posible (Feyman, 1959), ii) la invención y el desarrollo de instrumentos que nos permiten ver, medir y manipular átomos individuales (Binnig \& Rohrer, 1987), y iii) la conciencia de que la nanotecnología conduce a una amplia gama de nuevos desechos, tanto naturales como antropogénicos (Bhushan, 2004), con propiedades físicas y químicas sin precedentes, debido a efectos cuánticos.
Las aplicaciones de los nano-materiales y de los dispositivos nanoestructurados cubren un enorme número de campos, y continúan revolucionando nuestras vidas cotidianas en muchos aspectos fundamentales (Nanotechnology Benefits and Applications, 2016), de tal manera que este desarrollo también es denominado como "nano revolución "(CBC, 2017). Una visión general no exhaustiva de los campos de aplicación de NT que se muestra en la Fig. 1.

En la actualidad, los desarrollos de NT más impactantes a nivel mundial se realizan en las industrias de la microelectrónica y de la salud. La fabricación de circuitos electrónicos con dimensionamientos nanométricos al ritmo vertiginoso dictado por la Ley de Moore (Moore, 1965), ha revolucionado tanto la capacidad de cálculo de computadoras, pero también las

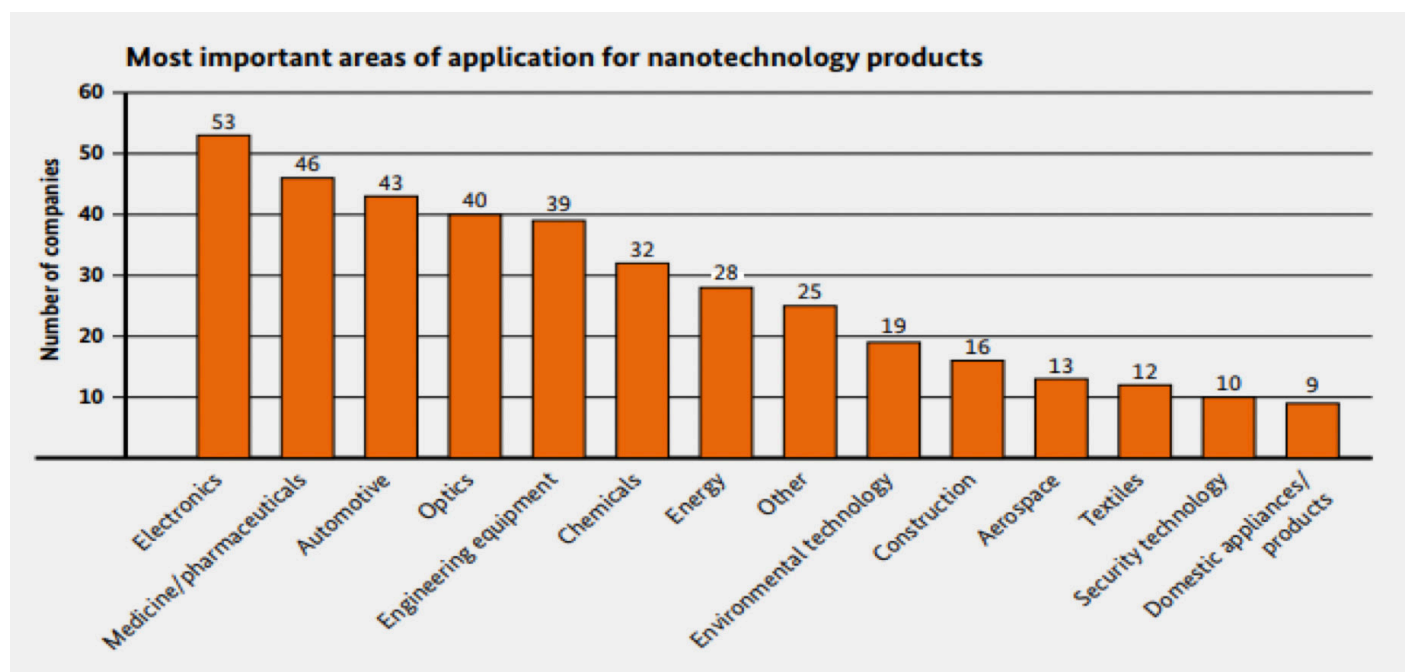

Fig. 1. Áreas de aplicación más importantes para los productos nanotecnológicos, según la encuesta de industriales participantes de 2013, VDI TZ, Alemania. (n=173, se permitió un máximo de 3 votos) Fuente: elaboración propia a partir de datos de (Federal Ministry of Education and Research, 2013). 
telecomunicaciones, con resultados difícilmente imaginables hace una década.

La nano-medicina (Nanomedicine Applications, 2018) proporciona soluciones terapéuticas sin precedentes para el tratamiento de enfermedades graves como el cáncer, la arterioesclerosis, el Alzheimer y la diabetes. Otras contribuciones de la nanomedicina incluyen sistemas avanzados de administración de medicamentos y tecnologías de reparación de tejidos, dispositivos de diagnóstico de alta resolución y la provisión de interfaces neuro-electrónicas mejoradas.

Sin embargo, los desarrollos arriba escritos en su mayoría son realizados en países con economías de escala, que cuentan con los conocimientos especializados, infraestructura muy avanzada, pero también el acceso "garantizado" a los mercados necesarios para comercializar la mayoría de este tipo de innovaciones. Estas condiciones no son dadas para la mayoría de los países centroamericanos, donde el enfoque de las Nanotecnologías tiene que ser diferente, y debería concentrarse en aplicaciones a temas vitales como las industrias de manufactura liviana y de servicios, pero también en alimentación, salud, vivienda, medio ambiente, e, importantemente educación, entre otras.

\section{Educación STEM}

El modelo de educación STEM (en inglés: Science, Technology, Engineering and Mathematics), acuñado por la Fundación Nacional de Ciencia (en inglés: National Science Foundation NSF) en los años 90 (Sanders, 2009), (Roco, Mirkin, \& Hersam, 2010), (Erdogan, Corlu, \& Capraro, 2013), (Stains et al., 2018), (Paredes,
Picardo, \& Torres, 2018) enfatiza una estrategia educativa interdisciplinaria, donde los conceptos académicamente rigurosos se acoplan a lo real; es decir, se ponen en práctica la ciencia, la tecnología, la ingeniería y las matemáticas en el contexto de solucionar problemas relacionados con la escuela, la sociedad, el deporte o el trabajo, entre otros(Jose, Lupiañez, 2016; Paredes et al., 2018).

Una importante componente del modelo de educación STEM incluye el Aprendizaje Activo Basado en Proyectos (Prince, 2004), que se desarrolla de forma colaborativa (Bruffee, 1995; Panitz, 2000) y cooperativa (Millis \& J. Cottell, 1998), formando equipos integrados por personas con perfiles diferentes, áreas disciplinarias, profesiones, idiomas y culturas que trabajan juntos para resolver problemas reales.

\section{Resultados}

A continuación, presentamos algunos resultados obtenidos mediante la aplicación consecuente de las metodologías anteriormente descritas.

\section{Desalinización de aguas marinas y purificación de aguas contaminadas}

Uno de los métodos de desalinización de aguas marinas más antiguos utilizados por el hombre (Ross, LL.D., \& Smith, 1931) y la naturaleza consiste en su destilación, usando el sol como fuente de energía.

Científicos del grupo de investigadores del MIT, USA (Ghasemi et al., 2014) han demostrado que la tasa de evaporación de agua puede aumentar considerablemente, hasta por un factor de 3, cuando una superficie húmeda 
expuesta a la irradiación contiene un material bidimensional con propiedades plasmónicas, con amplio espectro de absorción de luz visible, como por ejemplo el grafeno (Ye et al., 2013) o nanopartículas (Yu et al., 2015), (Wang, He, Liu, Cheng, \& Zhu, 2017), (Zhou et al., 2016).

En trabajos recientes (Christoph, Hernández, Muñoz, \& Ventura, 2016), (Hernández, Muñoz, Ventura, Büscher, \& Christoph, 2018), (Hernández et al., 2019), hemos demostrado el mismo efecto, i.e un $300 \%$ de incremento en la tasa de evaporación, utilizando componentes férricos de la arena negra, material que es abundante en la zona costera de Centroamérica.

Basado en este hallazgo, se han desarrollado tres prototipos con diferentes enfoques en el que se puede aplicar la tecnología de aceleración de evaporación, los cuales se denominan como: desalinizador clásico, flotante y parabólico (ver Fig. 2), para la fabricación y pruebas se trabaja en conjunto con estudiantes de la Universidad
Francisco Gavidia de El Salvador, quienes en su periodo de horas sociales participan activamente en este proyecto.

\section{Nanotecnología para aplicaciones agrícolas}

Se espera que la población de El Salvador actual de 6.4 millones de personas alcance los 7 millones en 2100 (United Nations, 2017). Esto implica que se necesitarán nuevos sistemas de producción de alimentos, agua y energía para garantizar la seguridad alimentaria. Por otro lado, la producción de más alimentos requiere recursos naturales, utilización de tierras, abastecimiento de agua y energía (C Glenn \& Florescu, 2016).

Esta realidad demanda a la investigación científica que aporte nuevos paradigmas y prácticas para resolver problemas básicos $\mathrm{y}$ elementales y a la vez complejos y diversos. Algunos ejemplos son los siguientes: (i) ¿Cómo alimentaremos a nuestros hijos? ii) ¿Cómo podemos aumentar simultáneamente

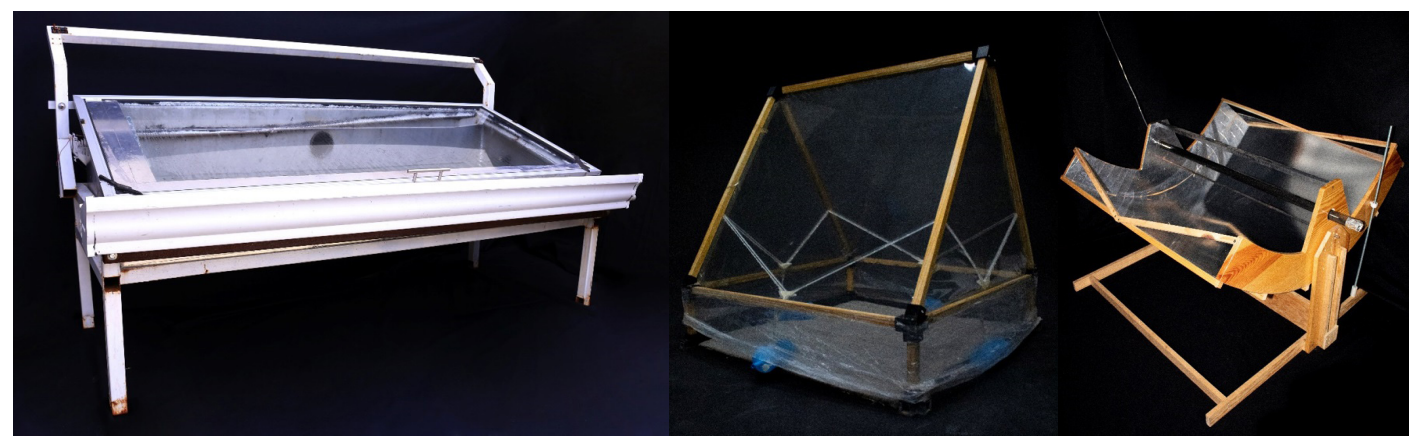

Fig. 2. Prototipos de desalinizadores solares realizados en UFG, E1 Salvador. Izquierda: Modelo clásico; basado en la literatura clásica del proceso de destilación. Centro: Modelo flotante; diseño de bajo costo para ser implementado directamente sobre la superficie de agua. Derecha: Modelo parabólico con alta concentración de luz para mejorar la eficiencia térmica del proceso de desalinización, que produce hasta 8 litros de agua desalinizada por día y metro cuadrado de superficie irradiada. 
el rendimiento de los cultivos y reducir el impacto ambiental de la agricultura? (iii) ¿Cómo contribuyen las plantas a los servicios de los ecosistemas (por ejemplo, la fotosíntesis, la fijación del nitrógeno y el ciclo de la materia orgánica) de los que depende la humanidad? ¿Podrán los sistemas agrícolas mundiales hacer frente al cambio climático mundial? (Chase et al., 2011), (de Oliveira, Campos, Bakshi, Abhilash, \& Fraceto, 2014).

La agricultura utiliza de manera ineficiente los insumos convencionales (tierra, agua, energía, fertilizantes y pesticidas), y una gran parte de los productos fitosanitarios aplicados al año se pierden o no están disponibles para el objetivo (DeRosa, Monreal, Schnitzer, Walsh, \& Sultan, 2010). Además, la agricultura (cultivos, ganadería y deforestación) es uno de los principales contribuyentes a las emisiones de gases de efecto invernadero y produce alrededor del $24 \%$ de la cantidad total anual a nivel mundial La producción de residuos es otro tema relevante del sector primario. Los países europeos producen aproximadamente 90 millones de toneladas de desechos agrícolas por año (Smith et al., 2014).

Entre las aplicaciones que se encuentran en estudio a nivel mundial bajo este sector tenemos:

- Nanosensores (Chhipa \& Joshi, 2016).

- Retención de agua (Ram, Vivek, \& Kumar, 2014).

- Desintoxicacion de suelo (Parisi, Vigani, \& Rodríguez-Cerezo, 2015), (Hasmaliza, Foo, \& Mohd, 2016).
- Control de enfermedades (Mishra et al., 2014), (Bramhanwade, Shende, Bonde, Gade, \& Rai, 2016).

- Nanoherbicidas (Ram et al., 2014), (Pérezde-Luque \& Rubiales, 2009).

- Nanopesticidas (Chhipa \& Joshi, 2016).

- Nanofertilizantes (Chhipa \& Joshi, 2016), (Duhan et al., 2017).

- Superficies foto-catalíticas (Fujishima \& Honda, 1972) (Bonetta, Bonetta, Motta, Strini, \& Carraro, 2013) (Giraldo, Franco, \& Arango, 2004) (Hasmaliza et al., 2016),

- Conservación de alimentos asequibles, mejora de la agricultura (Mousavi \& Rezaei, 2011), (Parisi, Vigani, \& Rodrigez-Cerezo, 2014)

En febrero 2019, la Universidad Francisco Gavidia inicia la investigación de aplicaciones nanotecnológicas para la agricultura de E1 Salvador, en el marco de una tesis de doctorado de Ing. Ángel Hernández. La investigación se concentra en la síntesis de hidrogeles, los cuales permiten prolongar el almacenamiento de agua en el tiempo, por lo cual es posible mantener húmeda la tierra de manera que una planta pueda hidratarse cuando lo necesita (Abobatta, 2018), (Tomczykowa \& PlonskaBrzezinska, 2019). Los geles son sistemas que se obtienen generalmente dispersando polímeros en solventes siendo los más utilizados agua y soluciones hidroalcohólicas.

Un hidrogel compuesto a base de poliacrilato de potasio es capaz de retener hasta 300 veces su 
peso en agua, producto de su descomposición genera potasio, dióxido de carbono, agua, etc (Liu \& Chan, 2015). La investigación también conlleva el uso de nutrientes mezclados en el hidrogel para propiciarle a la planta un mejor desarrollo, y evitando el desperdicio de nutrientes no focalizados mediante procesos de abonamiento convencionales (ver Fig. 3).

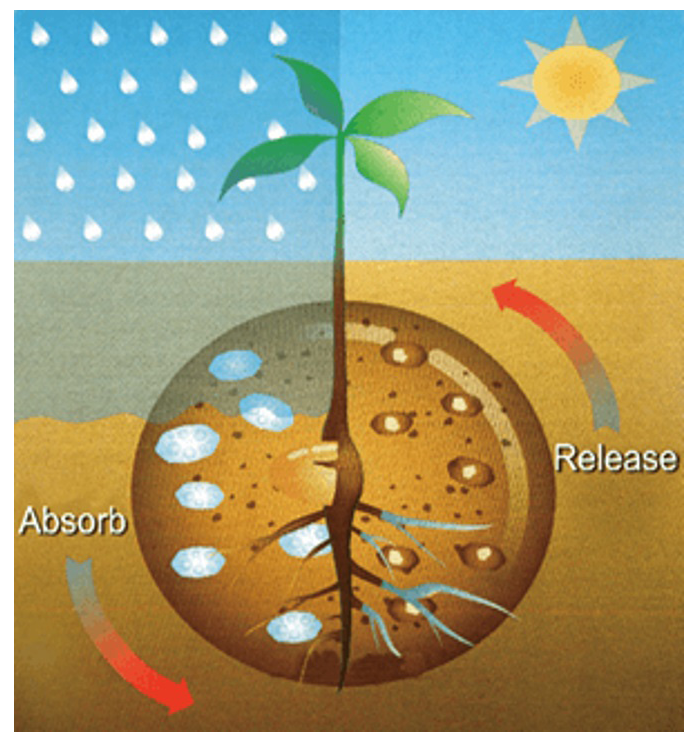

Fig. 3. El funcionamiento de un hidrogel es captar el agua lluvia y almacenarla durante épocas de sequía, y liberar el agua gradualmente. Fuente: (Graciela, 2019).

\section{Enfriamiento pasivo radiativo}

En la actualidad existen muchos equipos de refrigeración que propulsados con electricidad que permiten tener cámaras muy frías para almacenar alimentos, pero también enfriar el ambiente de casas, edificios y medios de transporte. Sin embargo, el costo energético, y por ende el impacto ambiental de estos equipos es relativamente alto.
En estos sistemas de enfriamiento convencionales, un objeto es enfriado utilizando procesos de transmisión convectivos y conductivos propulsados mediante acción mecánica para trasladar su calor hacia su entorno inmediato. E1 enfriamiento radiativo, aplicado en el desierto de Irán desde inicios del siglo XVII (Hosseini \& Namazian, 2012), (Fordham, 2018) sin embargo, prescinde de estos sistemas mecánicos activos y el calor es transmitido directamente al espacio exterior de la tierra, mediante transmisión radiativa de ondas electromagnéticas con longitudes en el rango entre 8,000 y 13,000 nanómetros, que no son absorbidas por la atmósfera terrestre. A este rango también se le conoce como "ventana atmosférica” (ver Fig. 4).

El espacio exterior de la atmósfera terrestre cuenta con temperaturas muy bajas (-273.14 ${ }^{\circ} \mathrm{C}$ ), que difieren por mucho de las temperaturas del planeta (entre $+17 \mathrm{a}+31^{\circ} \mathrm{C}$ en El Salvador). Esta diferencia de temperatura permite una transmisión de energía de forma muy efectiva para enfriar una superficie de un material que emita energía a través de la ventana atmosférica. La posibilidad de poder enviar calor excedente directamente al espacio, también abre nuevas expectativas en el manejo del calentamiento global (A. Raman, 2018).

La capacidad de emisión de luz es una de las propiedades fundamentales de la materia y las características del espectro emitido depende de las características de su composición y estructura atómica. En estudios recientes realizados por investigadores de Stanford University, USA (Rephaeli, Raman, \& Fan, 2013), (A. P. Raman, Anoma, Zhu, Rephaeli, \& Fan, 2014), se ha logrado sintetizar 


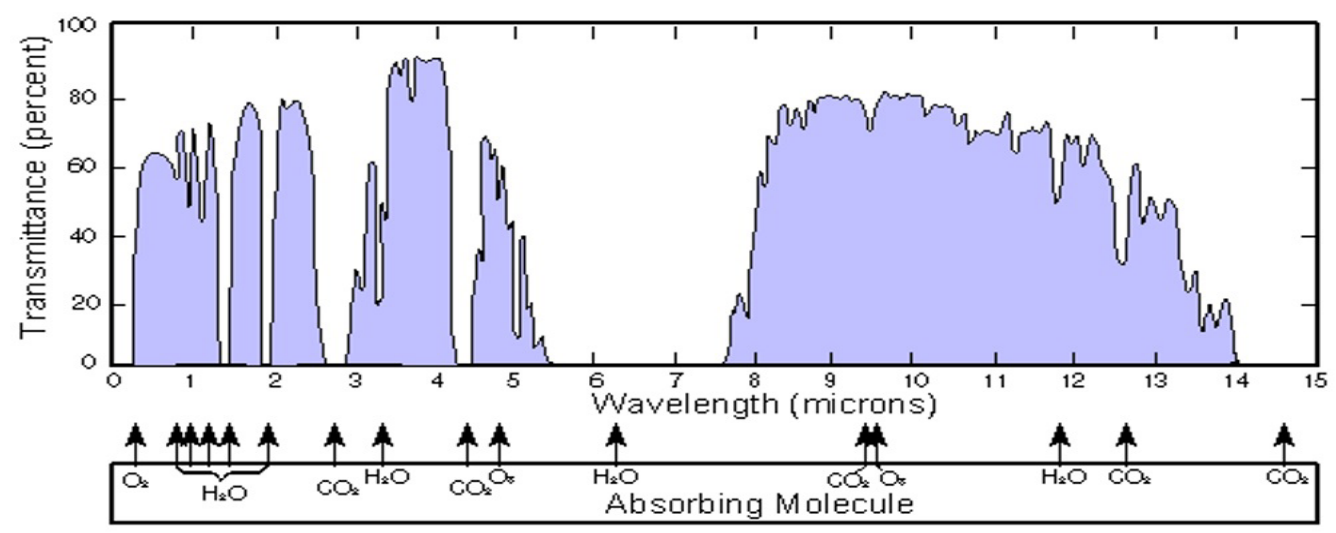

Fig. 4. Ventana atmosférica. Es el rango de longitudes de ondas electromagnéticas que puede atravesar los gases de la atmosfera sin obstáculo y por lo tanto alcanzar el espacio exterior con facilidad, este rango se define entre los 8 y los 13 micrómetros (8,000-13,000 nanómetros). Fuente: elaboración propia a partir de datos de (NASA, 2002)

combinaciones de estratos de diferentes materiales, utilizando métodos avanzados de epitaxia con haces moleculares, logrando obtener superficies que se "auto-refrigeran" con una potencia superior a 100 Watt por metro cuadrado de superficie expuesta.

La investigación doctoral recientemente iniciada en la Universidad Francisco Gavidia de E1 Salvador, por el Ing. Romeo Muñoz: "Caracterización de Materiales Naturales para el Enfriamiento Radiativo" se centra en la identificación de materiales de procedencia local, cuyo espectro de emisión radiativo coincida con la ventana atmosférica. Actualmente se investigan opciones de este tipo de materiales procedentes componentes de rocas ígneas localmente disponibles. Para cuantificar los flujos de energía emitida, se están diseñando diferentes tipos de termómetros de ultra-alta precisión, basado en sensores piezoeléctricos y ópticos.

\section{Educación STEM}

Con el apoyo de la Agencia de los Estados Unidos para el Desarrollo Internacional (USAID), el Instituto de Ciencia, Tecnología e Investigación de la Universidad Francisco Gavidia de E1 Salvador desarrolló un proyecto de educación que benefició a tres centros escolares públicos de dicho país (Humberstone, Picardo, \& Christoph, 2016).

El proyecto constó de tres fases: formación de los docentes, equipamiento de un Laboratorio Experimental STEM Básico y desarrollo de actividades extracurriculares e investigación por parte de los estudiantes. El objetivo del proyecto fue disminuir los hechos de violencia en las escuelas beneficiadas por medio de actividades extracurriculares para incentivar la investigación y experimentación en las asignaturas de Ciencias y Matemáticas. 
La línea base del proyecto incluyó indicadores de acceso a la calidad de la educación y conocimiento de los niveles de violencia. La primera medición se realizó con los datos del cierre del año escolar 2016. Durante el año 2017 se capacitó a los docentes de las escuelas beneficiadas en las áreas de: Metodología STEM, Planificación didáctica, Estadística aplicada, Programación, Base de datos, entre otros. Para incentivar la educación práctica se donó a las escuelas beneficiadas kits educativos de ciencias básicas, robótica e impresoras 3D para equipar a tres Laboratorios Experimentales STEM Básicos. Al finalizar el año escolar 2017 se realizó la segunda medición de los indicadores.

A continuación, resumimos algunos resultados obtenidos:

- Incremento de la calidad educativa: promedio de nota PAES, de 4.72 a 5.12 .

- Acceso a la educación: los resultados son favorables; ha disminuido la repitencia que genera frustración y deserción; reducción de la deserción escolar (-10\%).

- Violencia: Disminuyeron las intervenciones de la policía (-40\%).

- Durante los años 2018 y 2019 se brindó capacitación STEM, tanto teórica como práctica a más de 1500 estudiantes provenientes de la UFG, así como de otras instituciones educativas de El Salvador.

\section{Conclusiones}

Los resultados positivos obtenidos mediante una combinación de los innovadores enfoques combinados de Nanotecnología y STEM, dedicados a la búsqueda de soluciones específicas para mejorar la cobertura de las necesidades básicas de la población de una región determinada con los recursos localmente disponibles, motivan a considerar una ampliación de este concepto de investigación y educación multidisciplinaria y enfocada, con los siguientes componentes:

- Evaluar la factibilidad de la incorporación de propuestas y soluciones nanotecnológicas en los aspectos sociales y humanísticos, para también impulsar la proliferación de las mismas.

- Impulsar un programa para crear una red de Escuelas Superiores de Investigación Aplicada (ESCAs), con estructuras afines a un concepto modular y flexible, que permita enfocar y adaptar a los contendidos de $\mathrm{I}+\mathrm{D}$ y educativos las necesidades básicas de la población de sus regiones.

- Aplicar dicho programa a nivel nacional, pero también a nivel Mesoamericano y en otras regiones del mundo, donde amplios sectores de la población se ven confrontados con la triste necesidad de migrar, por no poder cubrir, de manera sostenible, sus necesidades básicas mediante esfuerzo propio.

\section{Bibliografía}

Abobatta, W. (2018). Impact of hydrogel polymer in agricultural sector. Advances in Agriculture and Environmental Science: Open Access (AAEOA), 1(2), 59-64. https://doi.org/10.30881/ aaeoa.00011 
Banco Interamericano de Desarrollo (BID). (2016). América Latina y el Caribe 2030: Escenarios futuros - See more at: https:// publications.iadb.org/handle/11319/7978\#sthash. eCRsB5D9.dpuf. 156. https://doi.org/http:// dx.doi.org/10.18235/0000535

Bhushan,B. (2004). Introduction to Nanotechnology. https://doi.org/10.1016/j.pragma.2005.06.002

Binnig, G., \& Rohrer, H. (1987). Scanning Tunneling Microscopy-from Birth to Adolescence (Nobel Lecture). Angerwandte Chemie International Edition in English, 26(7), 606-614. https://doi.org/10.1002/anie.198706061

Bonetta, S., Bonetta, S., Motta, F., Strini, A., \& Carraro, E. (2013). Photocatalytic bacterial inactivation by $\mathrm{TiO} 2$-coated surfaces. $A M B$ Express, 3(1), 59. https://doi.org/10.1186/21910855-3-59

Bramhanwade, K., Shende, S., Bonde, S., Gade, A., \& Rai, M. (2016). Fungicidal activity of $\mathrm{Cu}$ nanoparticles against Fusarium causing crop diseases. Environmental Chemistry Letters, 14(2), 229-235. https://doi.org/10.1007/s10311-0150543-1

Bruffee, K. A. (1995). Sharing Our Toys: Cooperative Learning Versus Collaborative Learning. Change: The Magazine of Higher Learning, 27(1), 12-18. https://doi.org/10.1080 /00091383.1995.9937722

C Glenn, J., \& Florescu, E. (2016). 2015-16 State of the Future. Journal of Socialomics, 5(3). https://doi.org/10.4172/2471-8726.1000168 CBC. (2017). The Nano Revolution: More than Human. Retrieved August 16, 2018, from http://www.cbc.ca/natureofthings/episodes/ the-nano-revolution-more-than-human

Chase, M., Clarke, M., Grierson, C., Grierson, D., Edwards, K., \& Jellis, G. (2011). Letters One hundred important questions facing plant science research. New Phytologist, 192, 6-12.

Chhipa, H., \& Joshi, P. (2016). Nanofertilisers, Nanopesticides and Nanosensors in Agriculture. In S. Ranjan, N. Dasgupta, \& E. Lichtfouse (Eds.), Nanoscience in Food and Agriculture 1 (pp. 247-282). https://doi.org/10.1007/978-3-31939303-2_9

Christoph, R., Hernández, A., Muñoz, R., \& Ventura, S. (2016). Estudios de materiales grafíticos comunes como aceleradores de evaporación de agua bajo irradiación luminosa. Boletín Nanotecria, September. Retrieved from http://nanotecnialab.ufg.edu.sv/frm/newsletters/ Nanoboletin_Septiembre-2016.pdf

De Oliveira, J. L., Campos, E. V. R., Bakshi, M., Abhilash, P. C., \& Fraceto, L. F. (2014). Application of nanotechnology for the encapsulation of botanical insecticides for sustainable agriculture: Prospects and promises. Biotechnology Advances, 32(8), 1550-1561. https:// doi.org/10.1016/J.BIOTECHADV.2014.10.010

DeRosa, M. C., Monreal, C., Schnitzer, M., Walsh, R., \& Sultan, Y. (2010). Nanotechnology in fertilizers. Nature Nanotechnology, 5(2), 9191. https://doi.org/10.1038/nnano.2010.2

Dowling, a, Clift, R., Grobert, N., Hutton, D., Oliver, R., O'neill, O., ... Et Al. 
(2004). Nanoscience and nanotechnologies: opportunities and uncertainties. London The Royal Society The Royal Academy of Engineering Report, 46(July), 618-618. https://doi. org/10.1007/s00234-004-1255-6

Duhan, J. S., Kumar, R., Kumar, N., Kaur, P., Nehra, K., \&Duhan, S. (2017). Nanotechnology: The new perspective in precision agriculture. Biotechnology Reports, 15(December 2016), 1123. https://doi.org/10.1016/j.btre.2017.03.002

Erdogan, N., Corlu, M. S., \& Capraro, R. M. (2013). Defining Innovation Literacy: Do Robotics Programs Help Students Develop Innovation Literacy Skills? International Online Journal of Educational Sciences, 5(1), 1-9.

Federal Ministry of Education and Research, G. (2013). nano.DE-Report 2013.

Feyman, R. F. (1959). Feynman's Talk. Retrieved August 16, 2018, from http://www. zyvex.com/nanotech/feynman.html

Fordham, M. (2018). THE PHYSICS OF FREEZING AT THE IRANIAN YAKHCHAL. Retrieved June 28, 2019, from https://www.maxfordham.com/researchinnovation/the-physics-of-freezing-at-theiranian-yakhchal/

Fujishima, A., \& Honda, K. (1972). TiO2 photoelectrochemistry and photocatalysis. Nature, 213(1998), 8656. Retrieved from https://electrochem.org/d1/ma/203/pdfs/2729. pdf\%5Cnpapers $2: / /$ publication/uuid/ F6B805BA-46E0-443A-B666-4F5841D3D337
Ghasemi, H., Ni, G., Marconnet, A. M., Loomis, J., Yerci, S., Miljkovic, N., \& Chen, G. (2014). Solar steam generation by heat localization. Nature Communications, 5, 1-7. https://doi.org/10.1038/ncomms5449

Giraldo, L. F. G., Franco, E. A. M., \& Arango, J. J. S. (2004). La fotocatálisis como alternativa para el tratamiento de aguas residuales. Revista Lasallista de Investigación, 1(1), 83-92.

Graciela. (2019). Cómo Hacer Y Utilizar Polímeros De Hidrogel Para Retener La Humedad En E1 Suelo. Retrieved July 25, 2019, from Cámara de agricultura website: http:// www.agroecuador.org/index.php/blog-noticias/ item/25-como-hacer-y-utilizar-polimeros-dehidrogel-para-retener-la-humedad-en-el-suelo

Hasmaliza, M., Foo, H. S., \& Mohd, K. (2016). Anatase as Antibacterial Material in Ceramic Tiles. Procedia Chemistry, 19, 828-834. https:// doi.org/10.1016/j.proche.2016.03.109

Hernández, A., Muñoz, R., Ventura, S., Büscher, W., \& Christoph, R. (2018). Study of low cost materials for the enhancement ofsolar seawater desalination. Periodico Tche Quimica, 15(29).

Hernández, A., Christoph, R., López, D. E., Rodríguez, A. C., Gavidia, F., \& Salvador, E. (2019). Enhanced Solar Desalination of Seawater through the use of Igneous Rock Compounds. Unpublished.

Hosseini, B., \& Namazian, A. (2012). An overview of Iranian ice repositories, an example of traditional indigenous architecture. Metu Journal of the Faculty of Architecture, 29(2), 223-234. https://doi.org/10.4305/METU.JFA.2012.2.10 
Humberstone, J., Picardo, O., \& Christoph, R. (2016). Data Sciences Training and Research to Address Crime and Insecurity in El Salvador. (August). Retrieved from http://www.peerstem.edu.sv/index.en.html

Lupiañez, J. (2016). Diseño de tareas para el desarrollo de la competencia STEM: los problemas de modelización matemática. Retrieved June 26, 2019, from http://www. educacontic.es/blog/diseno-de-tareas-parael-desarrollo-de-la-competencia-stem-losproblemas-de-modelizacion

Liu, X., \& Chan, Z. (2015). Application of potassium polyacrylate increases soil water status and improves growth of bermudagrass (Cynodon dactylon) under drought stress condition. Scientia Horticulturae, 197, 705-711. https:// doi.org/10.1016/J.SCIENTA.2015.10.041

Millis, B., \& J. Cottell, P. (1998). Cooperative Learning for Higher Education Faculty.

Mishra, S., Singh, B. R., Singh, A., Keswani, C., Naqvi, A. H., \& Singh, H. B. (2014). Biofabricated Silver Nanoparticles Act as a Strong Fungicide against Bipolaris sorokiniana Causing Spot Blotch Disease in Wheat. PLOS ONE, 9(5), e97881. https://doi.org/10.1371/ journal.pone.0097881

Moore, G. E. (1965). The density of transistors assembled on a micro chip doubles every 12 month. Electronics, 38(8). https://doi. org/10.1109/JPROC.1998.658762

Mousavi, S. R., \& Rezaei, M. (2011). Nanotechnology in Agriculture and Food
Production. J. Appl. Environmental Sci., (January), 414-419.

Nanomedicine Applications.

(2018).

Retrieved October 23, 2018, from https:// etp-nanomedicine.eu/about-nanomedicine/ nanomedicine-applications/

Nanotechnology Benefits and Applications. (2016). 1-8. Retrieved from https://www.nano.gov/ you/nanotechnology-benefits

NASA. (2002, September 17). Remote Sensing.

Organización de las Naciones Unidas para la Alimentación y la Agricultura-FAO-, OPS, WFP, OMS, \& UNICEF. (2018). Panorama de la seguridad alimentaria y nutricional en américa latina y el caribe, desigualdad y sistemas alimentarios. Panorama de La Seguridad Alimentaria, 133. https://doi.org/10.1111/ j.1365-2036.2010.04268.x

Panitz, T. (2000). Collaborative Versus Cooperative Learning: Comparing the Two Definitions Helps Understand the nature of Interactive learning. Cooperative Learning and College Teaching, 8(2), 13. Retrieved from https://goo.gl/CdQGL9

Paredes, M., Picardo, O., \& Torres, B. C. (2018). El modelo STEM. In Universidad Francisco Gavidia.

Parisi, C., Vigani, M., \& Rodrigez-Cerezo, E. (2014). Proceedings of a workshop on " Nanotechnology for the agricultural sector: from research to the field." https://doi. org/10.2791/80497 
Parisi, C., Vigani, M., \& Rodríguez-Cerezo, E. (2015). Agricultural Nanotechnologies: What are the current possibilities? Nano Today, 10(2), 124-127. https://doi.org/10.1016/j. nantod.2014.09.009

Pérez-de-Luque, A., \& Rubiales, D. (2009). Nanotechnology for parasitic plant control. Pest Management Science, 65(5), 540-545. https:// doi.org/10.1002/ps.1732

Ram, P., Vivek, K., \& Kumar, S. P. (2014). Nanotechnology in sustainable agriculture: Present concerns and future aspects. African Journal of Biotechnology, 13(6), 705-713. https:// doi.org/10.5897/AJBX2013.13554

Raman, A. (2018). How we can turn the cold of outer space into a renewable resource. Retrieved from https://www.ted.com/talks/ aaswath_raman_how_we_can_turn_the_cold_ of_outer_space_into_a_renewable_resource/ transcript?language $=$ en\#t -790722

Raman, A. P., Anoma, M. A., Zhu, L., Rephaeli, E., \& Fan, S. (2014). Passive radiative cooling below ambient air temperature under direct sunlight. Nature, 515(7528), 540-544. https://doi.org/10.1038/nature13883

Rephaeli, E., Raman, A., \& Fan, S. (2013). Ultrabroadband Photonic Structures To Achieve High-Performance Daytime Radiative Cooling. Nano Letters, 13(4), 1457-1461. https://doi. org/10.1021/nl4004283

Roco, M. C., Mirkin, C. A., \& Hersam, M. C. (2010). Nanotechnology research directions for societal needs in 2020 - Retrospective and
Outlook. WTEC-World Technology Evaluation Center, 476-477. https://doi.org/10.1007/97894-007-1168-6

Rodger W. Bybee. (2013). The Case for STEM Education: Challenges and Opportunities (illustrate). NSTA Press.

Ross, W. D., LL.D., \& Smith, J. A. (1931). Aristotele's METEOROLOGICA translated.

Sanders, M. (2009). STEM, STEM Education, STEMmania. THE TECHNOLOGY TEACHER, 41(1), 49-52.

Smith, P., Bustamante, M., Ahammad, H., Clark, H., Dong, H., Elsiddig, E. A., ... Tubiello, F. N. (2014). Agriculture, Forestry and Other Land Use (AFOLU) Coordinating Lead Authors: Lead Authors. Climate Change 2014: Mitigation of Climate Change. Contribution of Working Group III to the Fifth Assessment Report of the Intergovernmental Panel on Climate Change, 811-922. Retrieved from https://www.ipcc.ch/ pdf/assessment-report/ar5/wg3/ipcc_wg3_ar5_ chapter11.pdf

Stains, M., Harshman, J., Barker, M. K., Chasteen, S. V., Cole, R., DeChenne-Peters, S. E., ... Young, A. M. (2018). Anatomy of STEM teaching in North American universities. Science, 359(6383), 1468-1470. https://doi. org/10.1126/science.aap8892

Tomczykowa, M., \& Plonska-Brzezinska, M. E. (2019). Conducting polymers, hydrogels and their composites: Preparation, properties and bioapplications. Polymers, 11(2). 
United Nations. (2017). World Population Yu, S., Zhang, Y., Duan, H., Liu, Y., Quan, X., Prospects 2017 Revision. 53. Tao, P., ... Deng, T. (2015). The impact of surface chemistry on the performance of localized solarWang, X., He, Y., Liu, X., Cheng, G., \& Zhu, driven evaporation system. Scientific Reports, 5, 13600. https://doi.org/10.1038/srep13600

bio-inspired interface heating of broadbandabsorbing plasmonic membranes. Applied Energy, 195, 414-425. https://doi.org/10.1016/j. apenergy.2017.03.080

Ye, R., Xiang, C., Lin, J., Peng, Z., Huang, K., Yan, Z., ... Tour, J. M. (2013). Coal as an Zhou, L., Tan, Y., Ji, D., Zhu, B., Zhang, P., $\mathrm{Xu}$, J., ... Zhu, J. (2016). Self-assembly of highly efficient, broadband plasmonic absorbers for solar steam generation. Science Advances, 2(4), e1501227. https://doi.org/10.1126/ sciadv.1501227 abundant source of graphene quantum dots. Nature Communications, 4, 2943. https://doi. org/10.1038/ncomms3943 